\section{The "swinging" squamocolumnar junction in esophagocardiac achalasia}

A 45-year-old woman was referred for evaluation of longstanding dysphagia to both solids and fluids, and progressive regurgitation over the past 10 years. Besides glycogenic acanthosis, upper gastrointestinal endoscopy was suggestive of esophageal dilation with a hypomotile contraction pattern. During careful observation with air insufflation, the narrowed esophagogastric junction failed to relax but could be passed easily, giving rise to a popping sensation ( Fig. 1). Likewise, on retroflexion the cardia appeared tight, with discrete tongues extending from the squamocolumnar junction ( $\bullet$ Fig. 2). To better assess the endoscopic characteristics of the presumed achalasic cardia, we carried out a thorough dynamic retroflexed examination with different levels of scope insertion. In contrast with the close-up view, the esophagogastric junction sleeve flattened while advancing the endoscope, and the squamocolumnar junction was pulled out to come into full view ( Fig.3). This was reproducible on repetitive "to-and-fro" scope maneuvers, with the squamocolumnar junction swinging in and out of endoscopic view ( Video 1). The diagnosis of esophagocardiac achalasia was established by manometry, which confirmed resting lower esophageal sphincter (LES) hypertension $>60 \mathrm{mmHg}$ with lack of swallowinduced relaxation and low-amplitude simultaneous esophageal body contractions. The patient subsequently underwent uncomplicated pneumatic balloon dilation ( $30 \mathrm{~mm}$ Rigiflex II Single Use Achalasia Balloon Dilator; Boston Scientific, Ratingen, Germany).

Given that current practice trends in most countries prioritize upper gastrointestinal endoscopy in the diagnostic algorithm for patients with presumed esophageal dysphagia, the endoscopist should be aware of features suggestive of achalasia so as to

\section{Video 1}

The squamocolumnar junction swinging in and out of endoscopic view on repetitive "to-and-fro" scope maneuvers. avoid misdiagnosis, e.g., with strictures of peptic origin [1]. The clinical significance of endoscopy in achalasia is largely to rule out malignant pseudo-achalasia [2]. Despite endoscopy being negative in an estimated $40 \%-50 \%$ with less advanced or atypical motor disease, positive recognition of endoscopic achalasia characteristics is essential in stratifying patients for dedicated diagnostic work-up and treatment decisions [3]. The Identification of potential novel endoscopic markers of esophagocardiac achalasia, such as the as-yet unreported phenomenon of the "swinging" squamocolumnar junction, most likely reflecting resting LES hypertension, might be helpful in timely referral for specialized motility testing. However, the diagnostic accuracy of this endoscopic finding and its correlation with manometric findings warrants further assessment in systematic studies, e.g., by blinded videotape review.

Endoscopy_UCTN_Code_CCL_1AB_2AC_3AH

Competing interests: None

\section{Zimmer, F. Lammert}

Department of Medicine II, Saarland University Medical Center, Homburg, Germany

\section{References}

1 Kessing BF, Bredenoord AJ, Smout AJ. Erroneous diagnosis of gastroesophageal reflux disease in achalasia. Clin Gastroenterol Hepatol 2011; 9: 1020-1024

2 Kopelman Y, Triadafilopoulos G. Endoscopy in the diagnosis and management of motility disorders. Dig Dis Sci 2011; 56: 635-654

3 Francis DL, Katzka DA. Achalasia: update on the disease and its treatment. Gastroenterology 2010; 139: 369-374

\section{Bibliography}

DOI http://dx.doi.org/

$10.1055 / \mathrm{s}-0032-1308920$

Endoscopy 2012; 44: E201

(c) Georg Thieme Verlag KG

Stuttgart · New York

ISSN 0013-726X

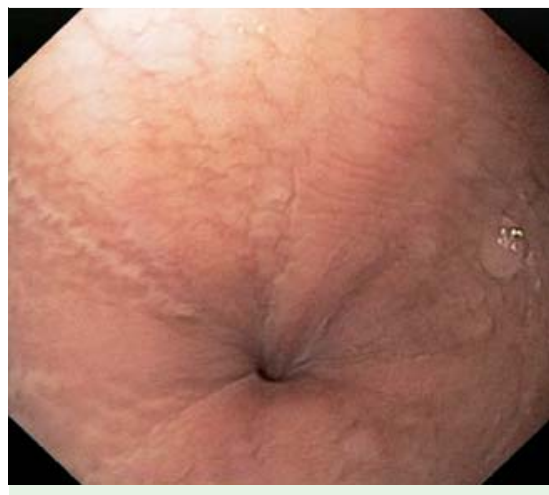

Fig. 1 Endoscopic view of the distal esophagus in a 45-year-old woman with long-standing dysphagia and progressive regurgitation showing a narrowed esophagogastric junction.

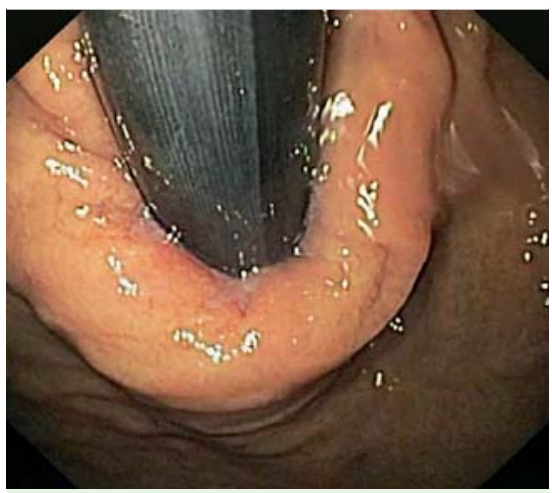

Fig. 2 Standard retroflexed view of the tightfitting cardia with discrete tongues of the squamocolumnar junction.

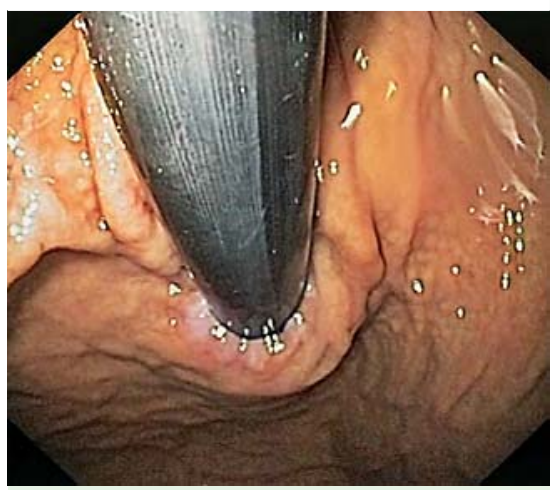

Fig.3 Maintained retroflexed view on deep scope insertion with the squamocolumnar junction coming into full view.

Corresponding author

\section{Dr V. Zimmer}

Department of Medicine II

Saarland University Medical Center

Kirrberger Str. 100

66421 Homburg

Germany

Fax: 0049-6841-1623264

vincent.zimmer@uks.eu 